\title{
Influencia del deterioro incipiente producido por el hongo de pudrición parda Serpula lacrymans, sobre las propiedades mecánicas de compresión normal y paralela a la fibra en madera de Pinus radiata D. Don
}

\author{
Influence of incipient decay caused by the brown-rot \\ fungy Serpula lacrimans, on the mechanical properties of \\ normal and parallel compression to the fiver in Pinus radiata \\ D. Don
}

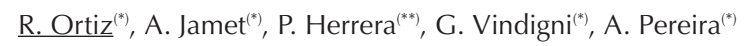

RESUMEN

Diversas edificaciones y estructuras construidas en madera son atacadas por hongos de pudrición, provocando en algunos casos, leves pérdidas de peso que se traducen en disminución en las propiedades resistentes del material.

En este trabajo se evaluó la influencia de estados incipientes de pudrición en madera, en las propiedades mecánicas de compresión paralela y compresión normal.

Para esto, se inocularon probetas de Pinus radiata $\mathrm{D}$. Don. con el hongo de pudrición parda Serpula lacrymans, las cuales fueron posteriormente ensayadas mecánicamente en tres diferentes períodos de exposición fúngica.

Los resultados obtenidos determinaron una importante disminución porcentual de las propiedades bajo estudio.

$660-9$

Palabras clave: Pinus radiata, pudrición, pérdida de peso, propiedades mecánicas, Serpula lacrymans.

\section{SUMMARY}

Several buildings and structures of wood are attacked by rot fungi, leading in some cases, slight weight losses that result in reductions in the strength properties of the material.

In this work was evaluated the influence of early rot decay in wood, in mechanical properties of normal and parallel compression.

For this, were inoculated samples of Pinus radiata $D$. Don with brown rot fungi Serpula Lacrymans. These samples were tested in three differents periods of fungal exposure.

The results identified a significant decrease in percentage of the properties under study.

Keywords: Pinus radiata, rot, weight loss, mechanical properties, Serpula lacrymans. 


\section{INTRODUCCION}

La madera está sujeta al ataque de hongos e insectos (1). Sin embargo, los principales daños son producidos por hongos filamentosos saprófitos (2-4). Estos hongos atacan la madera con diferentes grados de agresividad, modificando su apariencia, y produciendo daños significativos $(2,3,5)$.

El deterioro causado por hongos de pudrición parda resulta ser el más destructivo y mayoritario, debido a que puede causar rápidamente fallas estructurales (6). Entre estas clases de hongos se encuentra la especie Serpula Lacrymans, la cual es considerada como una de las más comunes en el daño de estructuras $(7,8)$, especie que ha producido, según Steenkjer et al. $(9,10)$, importantes pérdidas económicas en diferentes regiones del mundo tales como el norte y centro de Europa, Japón y Australia. Este tipo de hongo es considerado de pudrición seca, por lo que requiere contenidos de humedad sobre el $20 \%$, lo cual es levemente superior a la humedad que una estructura bien mantenida debiese tener (12-16\%) (8).

De acuerdo a lo señalado por Wilcox (11), los cambios en la composición química de la madera en estados tempranos de deterioro biológico causan considerable reducción de resistencia mecánica, antes de una evidente pérdida de peso. Clausen y Kartal (6) señalan que este tipo de daño se produce poco después de iniciada la colonización y la degradación enzimática, no existiendo evidencia visible. Por su parte, Zabel y Morrel (5) agregan que en estos estados ocurren leves cambios de color y textura, sin observar deterioro evidente del material.

Para la estimación de la disminución de resistencia en estados incipientes de deterioro de madera, se ha utilizado la evaluación mediante la pérdida de peso, lo que según Curling et al. $(12,13)$ es un buen indicador. Este método ha sido cuestionado por investigadores europeos, no obstante, se ha mostrado confiable con la mayoría de los hongos sometidos a estudio, siendo esta forma de medición la más ampliamente usada en el mundo (14). Highley (15) agrega que en estudios de laboratorio se ha encontrado que un $1 \%$ de pérdida de peso produce pérdidas en rigidez que varían entre un 6 y un $50 \%$, mientras que con un $10 \%$ de pérdida de peso la disminución de resistencia puede exceder el $50 \%$. Winandy y Morrel (16) estudiaron el efecto de deterioro fúngico sobre la resistencia mecánica en madera de Pseudotsuga menziesii, producida por los hongos de pudrición parda Postia placenta y GloeophyIlum trabeum, encontrando que pérdidas de peso entre $1 \%$ y $18 \%$, disminuyeron la resistencia entre $5 \%$ y $70 \%$. Curling et al. (12) desarrollaron un método de exposición fúngica que evaluó la resistencia mecánica en madera de Southern Yellow Pine colonizada por los hongos Postia placenta y Gloeophyllum trabeum y por el hongo de pudrición blanca Trametes versicolor. Los resultados obtenidos demostraron una directa relación entre pérdida de resistencia y pérdida de peso con ambos tipos de hongos de pudrición. Así mismo, señalaron que la disminución de resistencia muestra ser considerablemente más sensible que la pérdida de peso en estados incipientes de deterioro. Curlin et al. (17, 18) estudiaron el efecto de deterioro en la composición de hemicelulosas, en relación con las propiedades mecánicas en Southern Yellow Pine expuesto a deterioro por el hongo Gloeophyllum trabeum, determinando una relación de pérdida de resistencia/pérdida de peso $4 / 1$.

Winandy y Morrel (16) agregan que el deterioro temprano es importante en el uso estructural de la madera, debido al riesgo de colapso súbito del material en aparente buen estado. Bajo este contexto, Wilcox (11) afirma que el grado de deterioro puede variar grandemente en distancias cortas, siendo estos puntos débiles no conocidos, los que controlarán la capacidad final en servicio.

Con relación a lo anteriormente planteado, el objetivo de este estudio fue evaluar las propiedades mecánicas de compresión paralela y compresión normal a la fibra, en probetas a escala de Pinus radiata D. Don. inoculadas con el hongo de pudrición parda Serpula lacrymans, cuantificando su disminución mediante la determinación de la pérdida de peso en estados incipientes de pudrición.

\section{MATERIALES Y METODO}

\subsection{Madera}

Para el ensayo de pudrición se utilizaron probetas de madera de Pinus radiata D. Don., de $17 \times 17 \times 67 \mathrm{~mm}$ y de $25 \times 25 \times 75 \mathrm{~mm}$ correspondientes a los tamaños señalados por las normas NCh 973 Of. 86 (19) en escala 1:3 y NCh 974 Of. 86 (20) escala 1:2 respectivamente. La madera utilizada no presentó defectos como nudos, bolsas de resina, manchas y evidencia de ataque de microorganismos. Las probetas, secadas a $105 \pm 2{ }^{\circ} \mathrm{C}$ por 24 horas, fueron colocadas en un desecador por 30 minutos y luego pesadas con una precisión de 0,001 g. Posteriormente fueron sumergidas en agua por 2 horas con vacío, completando finalmente un total de 12 horas de inmersión, de manera de alcanzar una humedad de $110 \%$ para su posterior prueba de exposición fúngica. 


\subsection{Cepa y medios de cultivos}

Para la investigación se utilizó el hongo de pudrición parda Serpula lacrymans (Harm-888-R), proporcionado por el Laboratorio de Productos Forestales de Madison, Wisconsin, USA. La cepa fue mantenida en un medio de cultivo sólido preparado con agar 1,8\%, extracto de malta $1,5 \%$, fructosa $2 \%$, cloramfenicol 0,025 $\%$, sulfato de estreptomicina $0,025 \%$ y peptona $5 \%$, por litro de solución de agua destilada.

Trozos de agar con micelio del hongo fueron transferidos a 125 cc de medio de cultivo líquido el cual fue elaborado con los mismos nutrientes anteriormente señalados a excepción del agar. Estos trozos fueron cultivados a temperatura ambiente, en ausencia de luz y a 150 rpm por 3 días.

\subsection{Inoculación}

Frascos de vidrio de 1.000 cc fueron utilizados para el cultivo, dentro de los cuales se depositó sustrato de tierra de bosque considerando una relación suelo/aire 70/30, siendo humedecido según el factor de retención de agua (CRA). Se colocaron 5 probetas en cada frasco correspondiente al ensayo de compresión paralela, y 3 probetas en cada frasco de compresión normal, los cuales fueron esterilizados por un período de 60 minutos a una temperatura de $121^{\circ} \mathrm{C}$.

Las muestras de madera fueron inoculadas a través de medio líquido sumergiéndolas por 5 segundos, para posteriormente ser introducidas nuevamente en los frascos. Así mismo, probetas control de iguales características fueron sumergidas por 5 segundos en agua estéril. Una vez concluidos los períodos de incubación (4, 8 y 12 semanas) fueron extraídas de los frascos, limpiadas del micelio superficial y secadas en estufa a $105 \pm 2{ }^{\circ} \mathrm{C}$ por 24 horas. Posteriormente, fueron enfriadas en un desecador por 30 minutos y pesadas en balanza con precisión de 0,001 g, para determinar la pérdida de peso al final.

\subsection{Ensayos mecánicos}

Los ensayos mecánicos de las muestras fueron realizados conforme a lo establecido por la NCh 973 Of. 86 (19) para compresión para- lela, y NCh 974 Of. 86 (20) para compresión normal, mediante la utilización de una prensa hidráulica con precisión de carga de $1 \mathrm{~kg}$.

\section{RESULTADOS Y DISCUSION}

En todos los períodos de exposición fúngica, los resultados de comparación de medias t de Student entre la madera tratada y los controles, presentaron diferencias estadísticas significativas en las pérdidas de peso observadas para un nivel de confianza de 95\%. Las Tablas 1 y 2 muestran los resultados obtenidos en pérdidas de peso, y las disminuciones porcentuales del esfuerzo en el límite proporcional, esfuerzo en la carga máxima, y módulo elástico, para compresión paralela y normal respectivamente en las tres etapas de evaluación con respecto a la madera control.

Los resultados obtenidos en compresión paralela permitieron observar un aumento en la pérdida de peso en los 3 períodos de exposición fúngica, y cuyos valores se encuentran dentro de los rangos correspondientes a deterioro incipiente $(<10 \%)$. Así mismo, se determinó una importante disminución porcentual de los esfuerzos en el límite de proporcionalidad, carga máxima y módulo elástico, disminuyendo este último hasta cerca del $45 \%$ con respecto a la madera control, evidenciándose una progresiva e importante disminución de la rigidez del material y un aumento de las deformaciones a igual carga aplicada (Figura 1), aún cuando

\section{Gráfico Esfuerzo - Deformación} Compresión Paralela

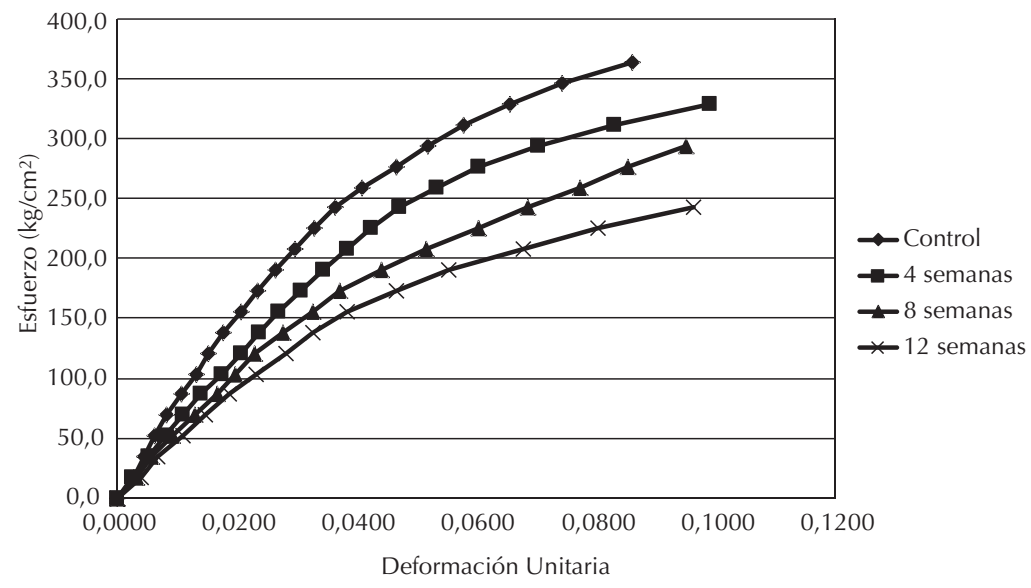

Tabla 1

Disminución porcentual de las propiedades mecánicas en compresión paralela

\begin{tabular}{|c|c|c|c|c|}
\hline $\begin{array}{c}\text { Período de } \\
\text { exposición } \\
\text { (semanas) }\end{array}$ & $\begin{array}{c}\text { Pérdida } \\
\text { de peso } \\
\%\end{array}$ & $\begin{array}{c}\text { Esfuerzo al } \\
\text { límite elástico } \\
\%\end{array}$ & $\begin{array}{c}\text { Esfuerzo } \\
\text { de rotura } \\
\%\end{array}$ & $\begin{array}{c}\text { Módulo de } \\
\text { elasticidad } \\
\%\end{array}$ \\
\hline 4 & 1,42 & 7,1 & 9,5 & 20,3 \\
\hline 8 & 2,91 & 21,4 & 25,1 & 35,3 \\
\hline 12 & 5,77 & 28,6 & 33,3 & 44,4 \\
\hline
\end{tabular}


no aparenta tener grandes cambios en sus características morfológicas, producto de la mínima degradación evidenciada por la acción del hongo en los rangos de pérdida de peso desarrolladas.

Con respecto a los resultados obtenidos en compresión normal, se observó un aumento de las pérdidas de peso en las tres etapas de exposición fúngica (Tabla 2), las cuales se encuentran dentro de los rangos correspon-
$42 \%$, con pérdidas de peso de $2 \%, 5 \%$ y $9 \%$, respectivamente. Toole (22) realizó extensos experimentos para determinar el efecto del deterioro producido por hongos de pudrición parda, sobre la resistencia a compresión perpendicular en maderas de coníferas, obteniendo disminución de resistencias de $18 \%, 24 \%$ y $48 \%$, con pérdidas de peso de $2 \%$, 3\% y $8 \%$, respectivamente. Así mismo, Clausen y Kartal (6) evaluaron la resistencia a compresión normal y el módulo de elas-

Tabla 2

Disminución porcentual de las propiedades mecánicas en compresión normal

\begin{tabular}{|c|c|c|c|c|}
\hline $\begin{array}{c}\text { Período de } \\
\text { exposición } \\
\text { (semanas) }\end{array}$ & $\begin{array}{c}\text { Pérdida de peso } \\
\%\end{array}$ & $\begin{array}{c}\text { Esfuerzo al } \\
\text { límite elástico } \\
\%\end{array}$ & $\begin{array}{c}\text { Esfuerzo de } \\
\text { rotura } \\
\%\end{array}$ & $\begin{array}{c}\text { Módulo de } \\
\text { elasticidad } \\
\%\end{array}$ \\
\hline 4 & 1,14 & 10 & 16,7 & 16,1 \\
\hline 8 & 2,86 & 20 & 29,2 & 41,1 \\
\hline 12 & 7,18 & 30 & 41,7 & 63,2 \\
\hline
\end{tabular}

\section{Gráfico Esfuerzo - Deformación} Compresión Normal

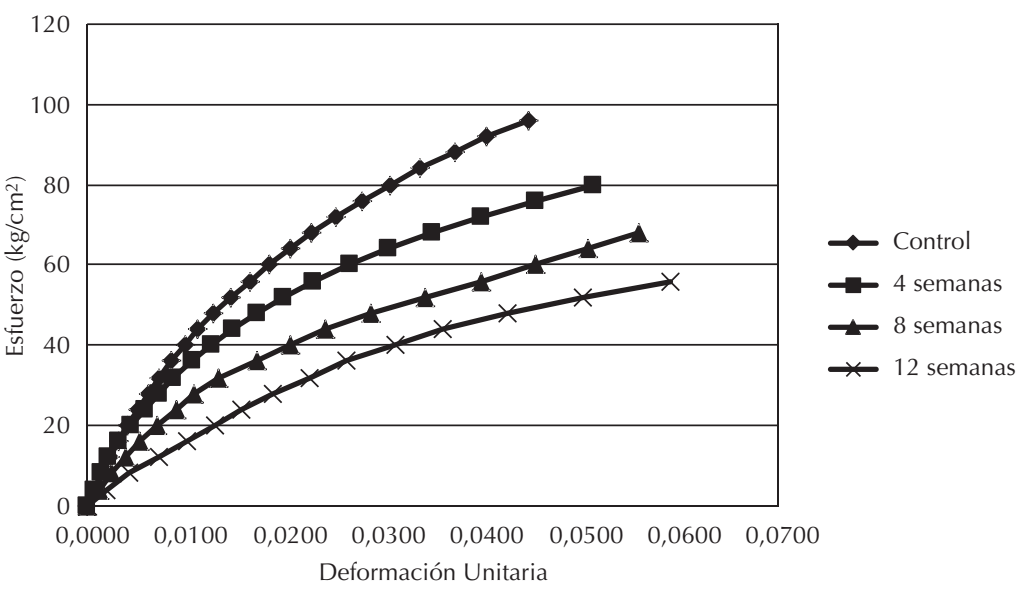

. Gráfico Esfuerzo deformación en compresión normal. dientes a deterioro incipiente $(<10 \%)$. Al igual que en compresión paralela, se determinó una importante disminución porcentual de los esfuerzos en el límite de proporcionalidad, carga máxima y módulo elástico, disminuyendo este último hasta cerca del $63 \%$ con respecto a la madera control. Así mismo, el material no evidenció cambio en su apariencia externa producto de la acción del hongo, no obstante, la disminución de su rigidez y el aumento de las deformaciones a igual carga aplicada quedo en evidencia (Figura 2).

La disminución de resistencia a compresión paralela a la fibra fue investigada por Mizumoto (21) en maderas de coníferas atacadas por hongos de pudrición parda, disminuyendo su valor en un $10 \%, 22 \%$ y ticidad, en bloques sometidos a la acción del hongo Postia Placenta, determinándose disminución de la resistencia a compresión en un $37 \%$ con un $9 \%$ de pérdida de peso. Al igual que los resultados de estas investigaciones, el presente trabajo determinó importantes reducciones en las resistencias a la compresión en ambos tipos de ensayos, comparable con los resultados obtenidos en maderas de coníferas sometidas al ataque de hongos de pudrición parda y bajo el $10 \%$ de pérdida de peso.

Blanchette et al. (23) señalan que los hongos de pudrición parda degradan los polisacáridos constituyentes de la pared celular mediante depolimerización sin extensiva pérdida de lignina. Cowling (24) agrega que los hongos de pudrición parda depolimerizan las holocelulosas, degradándose los productos más rápidamente que la forma que estos son consumidos. Zabell y Morrel (5) plantean que las hemicelulosas son a menudo las primeras componentes de la pared celular en ser degradadas, debido probablemente a sus cortas cadenas, solubilidad, y ubicación expuesta alrededor de las microfibrillas de celulosa. Esta depolimerización de los carbohidratos es reflejada según Green y Highley (25), en una rápida disminución de las propiedades resistentes de la madera. Winandy y Morrel (16) plantean que la degradación de hemicelulosas, tales como arabinosas y galactosas, juega un significativo rol en la disminución inicial de la capacidad resistente, agregando que las pérdidas de resistencia en madera y las pérdidas de peso producidas por hongos de pudrición parda están linealmente relacionadas. 


\section{CONCLUSIONES}

Como resultado de este trabajo se puede concluir lo siguiente.

Dentro de los rangos de pérdidas de peso obtenidos, se produjo una importante disminución de las propiedades mecánicas de compresión paralela y normal a la fibra, con respecto a las probetas control.

La condición de deterioro incipiente puede solamente ser considerada desde un punto de vista estético. Sin embargo, desde una visión estructural resulta fundamental para el caso de edificaciones u obras de infraestructura que consideren a la madera en algún elemento soportante y cuyas propiedades resistentes se encuentran drásticamente desmejoradas.

\section{AGRADFCIMIENTOS}

Los autores de este trabajo agradecen la colaboración brindada por el Laboratorio de Biodeterioro del Departamento de Ingeniería en Maderas de la Universidad del Bío Bío, Chile y la Carrera de Ingeniería en Construcción de DuocUC, Chile.

De igual manera se agradece la colaboración brindada por la Dirección de Investigación de la Universidad de Valparaíso DIPUV, en el financiamiento del proyecto 39/2008 "Metodología para la evaluación de la capacidad resistente de viviendas de madera en el casco histórico de Valparaíso mediante la técnica de ultrasonido".

\section{BIBLIOGRAFÍA}

(1) Yang, D.: "Potential utilization of plant and fungal extracts for wood protection". Forest Products Journal, Vol. 59 nº 4 (2009), pp. 97-103.

(2) Roff, J. W.; Csrjesi, A. J.; Swann, G. W.: "Prevention of sap stain and mold in packaged lumber", p. 43, Forintek Canad Corp., Western forest products laboratory. Technical report $n^{\circ} 14 R, 1980$.

(3) Blanchette, R.: "Delignification by Wood-decay fungi". Annu. Rev. Phytopathology, Vol. 29 (1991), pp 381-398. doi:10.1146/annurev.py.29.090191.002121

(4) Highley, T.; Dashek, W.: "Biotechnology in the study of brown and white rot decay", En: BRUCE, A y PALFERMAN, J. (Eds). Forest products biotechnology London, Great Britain: Taylor \& Francis, (1998) pp.15-36.

(5) Zabel, R.; Morrel, J. J.: "Wood microbiology: Decay and its prevention", p. 476, Academis Press, INC.USA, 1992.

(6) Clausen, C.; Kartal, S.: "Accelerated detection of brown-rot decay: Comparison of soil block test, chemical analysis, mechanical properties, and immunodetection", Forest Product Journal, Vol. 54 n 11-12 (2003), pp. 90-94.

(7) Singh, J.: "Fungal problems in historic buildings", Journal of architectural conservation, Vol. $6 n^{\circ} 1$ (2000).

(8) Singh, J.: "Designing out Timber Deterioration: Environmentally Sustainable Conservation Solutions", En: TIMBER IN the built heritage conference: 17-18 de Octubre de 2002. Edinburgo, Escocia: p. 11, 2002.

(9) Steenkjaer, A.; Green, F.; Clausen, C.; Jensen, B.: "The Dry Rot Fungus and Tolerance Towards Copper-Based Wood Preservaties", Serpula Lacrymans, En: 36th ANNUAL MEETING: The international research group on Wood protection: 24-28 de Abril de 2005. Bangalore, India: 2005. Documento IRG/WP 05-10555. p.7, 2005

(10) Steenkjaer, A.; Green, F.; Clausen, C.; Jensen, B.: "Tolerance of Serpula Lacrymans to copper-based wood preservaties". International Biodeterioration \& Biodegradation, Vol. 56 (2005), pp. 173-177. doi:10.1016/j.ibiod.2005.06.008.

(11) Wilcox, W.: "Review of literature on the effects of early stages on wood strength". Wood and fibers, Vol. $9 \mathrm{n}^{\circ} 4$ (1978), pp. 252-257.

(12) Curling, S.; Winandy, J. E.; Clausen, C. A.: "An experimental method to simulate incipient decay of wood by basidiomycete fungi", En: 31 st ANNUAL MEETING The International Research Group on Wood Preservation: 14-19 de Mayo de 2000. Kona, Hawaii. Stockholm, Suecia: Documento IRG/WP 00-20200. pp 1-12, 2000.

(13) Curling, S.; Clausen, C.; Winandy, J.: "Experimental method to quantify progessive stages of decay of wood by basidiomycete fungi". International Biodeterioration \& Biodegadation, Vol. 49 (2002), pp.13-19. doi:10.1016/S0964-8305(01)00101-9.

(14) Hartley, C.: "Evaluation of wood decay in experimental work", USDA Forest Service, Forest Products Laboratory, Mimeo n² 2119, 1958. 
(15) Highley, T.; Biodeterioration of wood Wood handbook: wood as an engineering material. Madison, WI: USDA Forest Service, Forest Products Laboratory, 1999. General technical report FPL ; GTR-113: Pages 13.1-13.16, 1999

(16) Winandy, J.; Morrel, J.: "Relationship between incipient decay, strength, and chemical composition of Douglas-fir heartwood". Wood and Fiber Science, Vol. 25 n³ (1993), pp. 278-288.

(17) Curling, S.; Winandy, J. E.; Clausen, C. A.: "The effect of hemicellulose degadation on the mechanical properties of wood during brown rot decay", En: 32nd ANNUAL MEETING The International Research Group on Wood Preservation: 20-25 de Mayo de 2001. Nara, Japón: Documento IRG/WP 01-20219. pp. 1-10, 2001.

(18) Curling, S.; Clausen, C.; Winandy, J.: "Relationships between mechanical propierties, weight loss, and chemical composition of wood during incipient brown-rot decay", Forest Product Journal, Vol. 52 nº7-8 (2002), pp.34-39.

(19) Norma Chilena, NCh 973 Of 86. Determinación de las propiedades mecánicas. Ensayo de compresión paralela a las fibras.

(20) Norma Chilena, NCh 974 Of 86. Determinación de las propiedades mecánicas. Ensayo de compresión perpendicular a las fibras.

(21) Mizumoto, S.: "The effect of decay caused by Gloeophyllum trabeum on the strength properties of Japanese red pine sap-wood". Journal of Japanese Forestry Society, Vol. 48 $\mathrm{n}^{\circ} 1$ (1966), pp. 7-11.

(22) Toole, E.: "Reduction in crushing strength and weight associated with decay by rot fungi". Wood Science, Vol. 3 no 3 (1971), pp. 172-178.

(23) Blanchette, R. A.; Nilsson, T.; Daniel, G.; Abad, A.: "Biological degradation of Wood" En: ROWELL, R.M. y BARBOUR, R.J. (Eds.), Archaeological Wood: Properties, Chemistry, and Preservation, Advances in Chemistry Series, Washington, DC. American Chemical Society, (1990) vol. 225 pp. 141-174.

(24) Cowling. E.: "Comparative biochemistry of decay of sweetgum sapwood by white rot and brown rot fungi" p. 79, Tech. Bull. No. 1258. U.S. Department of Agriculture, Washington. D.C., 1961.

(25) Green, F., Highley, T. "Mechanism of Brown-Rot Decay: Paradigm or Paradox". International Biodeterioration \& Biodegradation, Vol 39 n 2-3 (1997), pp. 113-124. doi:10.1016/ S0964-8305(96)00063-7. 